\title{
Power Aware Cluster Based Routing (PACBR) Protocol for Wireless Sensor Network
}

\author{
Ayan Kumar Das ${ }^{1}$, Rituparna Chaki ${ }^{2}$, and Atreyee Biswas ${ }^{3}$ \\ ${ }^{1}$ Department of Information Technology, Calcutta Institute of Engineering and Management, \\ Kolkata, India \\ ayandas24114057@yahoo.co.in \\ ${ }^{2}$ A.K. Chowdhury School of IT, University of Calcutta, \\ Kolkata, India \\ rituchaki@gmail.com \\ ${ }^{3}$ Department of Natural Science, West Bengal University of Technology, \\ Kolkata, India \\ atreyee11@gmail.com
}

\begin{abstract}
Energy efficiency is the main challenge of wireless sensor network (WSN). Many routing algorithms have been designed to meet the demand. Hierarchical clustering is one of the effective techniques for WSN to reduce the load in the network by data aggregation, thus saving energy. In this approach cluster heads take the responsibility to gather information of different events from its neighbors and send that to the sink node. Thus cluster heads loose its energy very early, which causes selection of new cluster head before starting of every round. This paper presents a multilevel cluster based protocol for energy efficient data communication. There is a cluster head selection phase followed by sensing of data, data gathering, aggregation and finally sending the aggregated data to the base station. Simulation results show that the performance of the proposed protocol is better than Energy Efficient Clustering Algorithm (EECA) for data aggregation in WSNs.
\end{abstract}

Keywords: Wireless Sensor Network, Data aggregation, Network Lifetime, Energy Efficiency, Cluster Head, Heterogeneous.

\section{$1 \quad$ Introduction}

A wireless sensor network consists of many small sensor nodes for sensing events in a particular area and sending that information to a sink node. The sensors are very small devices, with limited battery power, and often, with no source of recharge. This makes energy efficiency the main challenge for the researchers. Most of the power aware routing algorithms aim to find the best probable path to send the information between source and base station. Often, the sensor nodes are deployed very densely so that a number of nodes sense the same event. All these nodes try to send the redundant data to the sink node using multiple paths. This may cause a huge amount of energy drainage. Selection of a head node for a region or cluster can reduce the energy drainage in such situations. All the cluster members send the sensed data to the 
cluster head. The cluster head collects data from the members, reduce those redundant data and aggregate all the collected data. The aggregated data will be sent to the base station only by the cluster head, thus saving energy. The proposed algorithm selects a cluster head in different clusters before starting every round of aggregation and sends data to base station. The energy of the cluster head reduces, thus making the selection of new cluster head necessary after completion of every round. The energy consumption for communication between two nodes is governed by the Inverse Square Law of energy. Further the network is divided into different levels and every level has multiple clusters. All the aggregated packets coming from different cluster heads of higher level will be further aggregated by a leader node of lower level which is selected among the cluster heads of that level. Leader node will be selected depending on the distance from the base station and its residual energy. Finally the leader node will send the total aggregated packet to the leader node of next lower level and ultimately to the base station.

The remaining part of this paper is organized as follows: Section 2 deals with the review of state of the art routing topologies, section 3 gives a description of the proposed methodology, section 4 contains the simulation reports and section 5 is the concluding part.

\section{Review Works}

Energy Efficiency and increasing network longevity is the main research area in wireless sensor network for the last few years. To reduce energy drainage many algorithms have been designed to form clusters. A cluster head is selected to aggregate collected data and send that to the base station. Energy Efficient Clustering Algorithm for data aggregation in WSN [3] is one of the examples of clustering algorithm. It includes two phases of clustering. One is the formation of cluster heads. In this phase every node broadcast their radius, residual energy and co-ordinates to the neighbor nodes. Then the nodes will calculate competition bids to select the cluster head. The other phase is data aggregation and tree construction, which includes calculation of weight values for cluster heads depending on the distance from the base station and remaining residual energy. These weight values help to select the leader node among the cluster heads. The aggregated data will be sent to base station only by leader node which leads to uniform energy dissipation and long network longevity.

Another algorithm Energy Efficient Heterogeneous Clustered scheme for Wireless Sensor Network [5] has assumed that a percentage of sensor nodes are equipped with more energy and are immobile with known geographical locations. The introduction of computational heterogeneity includes more powerful microprocessor, more energy, complex data processing ability, which adds a lot of advantages to this model. The Link heterogeneity is introduced with the inclusion of high bandwidth and long distance network transceiver to prolong the lifetime of the network together with reliable data transmission. The Energy heterogeneity brought about the energy efficiency to the network, however increasing the implementation cost. 
Low Energy Adaptive Clustering Hierarchy (LEACH) $[1,2,11,20]$ is also clusterbased protocol, which includes distributed cluster formation. LEACH randomly selects a few sensor nodes as cluster heads (CHs). Then it rotates this role to evenly distribute the energy consumption among the sensors in the network. The cluster head $(\mathrm{CH})$ compress the collected data from different nodes that belong to the respective cluster. At last that the cluster head sends that aggregated packet to the base station in order to reduce the amount of information that must be transmitted to the base station. This protocol is most appropriate when there is a need for constant monitoring by the sensor network. A user may not need all the data immediately. Hence, periodic data transmissions are unnecessary which may drain the limited energy of the sensor nodes. After a given interval of time, a randomized rotation of the role of the $\mathrm{CH}$ is conducted so that uniform energy dissipation in the sensor network is obtained.

The Centralized approach on the other hand includes protocols such as LEACH-C $[1,2]$ which proposes the sending of location awareness of the nodes to the base station which in turn would select the cluster head on the basis of remaining energy. Such process is though effective on the basis of total energy dissipation, formation of correct number of cluster heads, the main disadvantage of this process lies in the overhead caused due to continuous involvement of the base station.

Hybrid Energy-Efficient Distributed (HEED) routing [8] is also a clustering approach, which is one of the most recognized energy-efficient clustering protocols. It extends the basic scheme of LEACH by using residual energy and node degree or density. In HEED, the initial probability for each node to become a tentative cluster head depends on its residual energy, and final heads are selected according to the intra-cluster communication cost. The clustering process is divided into a number of iterations, and terminates with in a constant number of iterations. HEED achieves fairly uniform distribution of cluster heads across the network.

PEGASIS [10] includes arranging of nodes into chains so that they can communicate only with their closest neighbor, thereby minimizing the power requirement for data transmission per second and reducing overhead. However failure of any intermediate node can cut off the link between other nodes and thus can detach a portion of the network.

Clustering and multi-hop routing with power control in Wireless Sensor Network provides clustering together with power control by means of algorithms like QFR and DCNPE [6], which are power efficient and thus prolongs the network lifetime. These algorithms state that the process of clustering is completed in two different phases, namely, THE SET UP PHASE, where the node with the maximum residual energy and maximum intra cluster broadcast power, is chosen as the cluster head; and THE STEADY STATE PHASE, where packets of request are send by the Base station through a proper power level, confirming that those packets never appear twice for a node. On receiving the request the sensor nodes send sensed data to the cluster head in a single hop; which in turn follows a reverse way to the path through which request came to it from the base station, to send the aggregated data to the Base station. The intra cluster communication process is same as LEACH whereas inter cluster communication takes place only between two cluster heads. The base station follows a 
power efficient path to send requests to the cluster head. Cluster heads follow the reverse path, by which energy of the nodes involved is reduced considerably.

QOS supporting and optimal energy allocation for a cluster based Wireless Sensor Network [7] states that together with energy efficiency, The Quality of Service, which includes source to link delay, data pass rate, data loss rate etc. must also be taken under consideration. The algorithm states that each cluster is controlled by a cluster head having a finite capacity called SINGLE FIXED RATE. The relaying of traffic from cluster to cluster till the sink to minimize the data congestion and increase network lifetime, makes the cluster heads depend on total relaying data rate from its own cluster as well as other clusters.

A novel hierarchical routing protocol algorithm for Wireless Sensor Networks [9] considers energy usage and packet latency together with security against node consideration attack. The simulation result proves that NHPRA can resist more against node consideration attack than LEACH due to the level maintained hierarchy adopted by them. The communication process among the sensors is same as GSPR, however the network is prone to outside node attack.

Energy Efficient Clustering under the joint Routing and coverage constraint [4] addresses optimal planning of the different states of sensors, providing energy efficient scheduling of the states, energy efficient routing, clustering and data aggregation. The algorithm formulates the problem as an ILP model and implementation of TABU search algorithm to manage exponentially increasing computation times. It mentions four different states of sensor node such as Transmit, Receive, Idle and Sleep. A subset of the total number of nodes will remain active at a time to save energy and reduce redundancy. The cluster heads are chosen dynamically on the basis of residual energy and distance from the neighbors and a spanning tree connects all cluster heads which are only capable of routing and thus send data to the sink. It is stated that all nodes have same sensing range and transmission range and the cluster heads are dynamically selected from the nodes.

\section{Proposed Work}

Most of the existing clustering algorithms send the aggregated packet to the base station from cluster head directly. As a result the energy of cluster head node goes down very quickly and it becomes non-functioning very soon. Some other algorithm like EECA [3] constructs a data aggregation tree where every sub cluster head sends the data to its parent node. The parent node contents of more weight value than its child. The weight value will be calculated depending on the remaining energy and distance from the base station. The problem with this algorithm is the child cluster head may send the aggregated data to its immediate parent node which belongs to the same level, instead of sending it to the next level directly. This leads to wastage of energy, delay and also reduces network lifetime. The proposed algorithm PACBR will divide the network into different levels, many clusters with cluster heads can be there in a same level, and sends the aggregated data to the base station in an energy efficient way and increases network lifetime also. 


\subsection{Basic Methodology}

The Power Aware Cluster Based Routing (PACBR) protocol for Wireless Sensor Network is proposed to send the information of devastating events from source node to base station. The algorithm clusterizes the sensor nodes and chooses a cluster head for every cluster. Every node will send the sensed data to its cluster head and it will take the responsibility to aggregate those data and send that to the base station via other cluster heads. The algorithm assumes that the network is a static network, that is, after deployment the nodes are motionless and contents of same initial energy. Base station or sink node will be of high configuration, i.e. contents of high energy, enough memory etc., deployed in a controllable place outside the network region. All sensor nodes are capable of data aggregation, compute its own residual energy and can find its geographic location.

All the sensor nodes send their geographic location to the base station which will group the sensor nodes into different clusters accordingly. The whole network will be divided in different levels and a level can have more than one clusters. The level nearest to base station will be the lowest level (i.e. level 1).

After deployment all the nodes will start to sense different events and send the sensed data to its cluster head. The cluster heads aggregate the data and send them to the sink node. A huge amount of energy is needed for this type of transmission which may cause early death of the cluster head. Thus for every round, change of cluster head is required. The algorithm helps to select the new cluster head and route the aggregated data to the base station.

The energy consumed in transmitting information from one node to another can be measured with the help of Inverse Square Law. It states that the intensity (energy per unit area perpendicular to the source) of linear waves radiating from a point source is inversely proportional to the square of the distance from the source. The intensity of energy for sending data from one node to another is thus -

$$
\mathrm{E}_{\mathrm{I}}=\frac{C}{(\text { distance })^{2}}
$$

Where $\mathrm{C}$ is a constant.

The consumption of energy will be increasing with the decrease in intensity. Thus energy consumed for a node $\mathrm{i}$ after sending $\mathrm{n}$ number of messages can be calculated as-

$$
\mathrm{E}_{\mathrm{ci}}=\sum_{j=1}^{n} \mathrm{k} * \mathrm{~d}_{\mathrm{ij}}^{2} * \mathrm{~N}_{\mathrm{m}}+\mathrm{E}_{\mathrm{a}}
$$

Where,

$$
\begin{aligned}
& \mathrm{d}=\text { Distance between nodes } \mathrm{i} \text { and } \mathrm{j} \\
& \mathrm{Nm}=\text { Total number of sent messages from node } \mathrm{i} \text { to } \mathrm{j} \\
& \mathrm{k}=\text { Constant value } \\
& \mathrm{E}_{\mathrm{a}}=\text { Energy required for data aggregation } \\
& \text { The residual energy for node } \mathrm{i} \text { will be- }
\end{aligned}
$$




$$
E_{R i}=E_{i}-E_{c i}
$$

If the residual energy $\mathrm{E}_{\mathrm{Ri}}$ of node $\mathrm{i}$ is greater than the threshold value then it becomes a candidate node of cluster head selection process. Threshold value can be defined as energy required to accept data from all nodes, aggregate that, and send that to neighbor nodes. Every candidate node will calculate the selection probability value Pi for itself.

$$
P_{i}=\frac{E_{R i}}{D_{i}}
$$

Where,

$$
D_{i}=\sum_{j=1}^{n} \mathrm{~d}_{\mathrm{ij}} / \mathrm{n}
$$

$E_{R i}$ is the remaining energy at node $i$ and $D_{i}$ is the average distance of that node from all its neighbor nodes lying in the same cluster. ' $n$ ' is the total number of neighbor nodes of node $i$ in that cluster.

Now all the nodes will broadcast their probability value $\mathrm{P}_{\mathrm{i}}$ to their neighbors. Every node will check the values and find the maximum. The node containing the maximum $\mathrm{P}_{\mathrm{i}}$ value will send the success message to all its neighbors.

A leader node is selected from among the cluster heads of the next lower level. The cluster head with highest weight value will selected as the leader node of that level. Every cluster head will try to send the aggregated data collected from different nodes to any of the cluster head of the next lower level. To select the cluster head of the next lower level it will calculate a weight value of those cluster heads by the following equation-

$$
W_{t}=K * \frac{E_{R}}{D_{B} * D_{N}}
$$

Where,

$\mathrm{E}_{\mathrm{R}}=$ Remaining energy of the cluster head of next lower level to which the current cluster head of higher level can send data.

$\mathrm{D}_{\mathrm{B}}=$ Distance from the base station of the cluster head of next lower level.

$\mathrm{D}_{\mathrm{N}}=$ Distance of the current cluster head from the cluster head of the next level to which it can send data.

$\mathrm{K}=$ constant

The cluster head with highest weight value shall be called leader node of that level. Thus the cluster head will send the aggregated data to the leader node of next lower level. Then that leader node will send that to its next lower level leader node and so on. The process continues until the aggregated data reached at lowest level (i.e. level 1). The cluster head of lowest level will not try to calculate any weight value and send the aggregated data to the base station directly. 


\subsection{Data Dictionary}

Table 1. Variables list

\begin{tabular}{|l|l|}
\hline \multicolumn{1}{|c|}{ Variable name } & \multicolumn{1}{c|}{ Description } \\
\hline $\mathrm{N}$ & Total Number of nodes \\
\hline $\mathrm{X}, \mathrm{y}$ & Coordinate values of every node \\
\hline $\mathrm{d}[\mathrm{i}][\mathrm{j}]$ & Distance between nodes $\mathrm{i}$ and $\mathrm{j}$ \\
\hline $\mathrm{P}[$ node_number] & An array consists probability values of each node \\
\hline $\mathrm{E}[$ node_number] & An array consists remaining energy of each node \\
\hline $\mathrm{E}_{\mathrm{Ci}}$ & Stores consumed energy for node $\mathrm{i}$ \\
\hline $\mathrm{D}_{\mathrm{i}}$ & Average distance of all neighbor nodes from node $\mathrm{i}$ \\
\hline
\end{tabular}

\subsection{Description of PACBR Algorithm}

1. Input geographic location (Two coordinate values $\mathrm{x}, \mathrm{y})$ of every node.

2. Make the cluster depending on the value of $\mathrm{x}$ and $\mathrm{y}$, and send the Cluster Information Packet (CIP) [Node ID, Cluster ID] to every node $\mathrm{N}_{i}$.

3. //Calculate energy consumed for every node iRepeat step 4 and 6 for $i=1$ to $n$

4. Repeat step 5 for $j=1$ to $k$

5. a) Read $\mathrm{Nm}$ and Ea b) $\mathrm{Eci}=\mathrm{ECi}+(\mathrm{c} * \mathrm{~d}[\mathrm{i}][\mathrm{j}] * \mathrm{Nm})+\mathrm{Ea}$

/ / End of Inner loop

6. //Calculate Residual Energy Set $E[i]=E[i]-E C i$

//End of outer loop

7. For $i=1$ to $n$ repeat step 8

8. If $E[i]>$ Eth Then

a. Set $S[i]=1$

b. For $j=1$ to $n$ repeat step $c$

c. Set $D=D+d[i][j]$ //End of loop

d. Set $D i=D / n$

e. Set $P[i]=E[i] / D i$ Else

//End of Forlooop

$$
\text { Set } S[i]=0, \quad P[i]=0
$$

9. //Broadcast probability value of every node to its neighbor within cluster only

Call Prob_Broadcast (P [ ], n)

10. //Find the max value among the received probability values

Call Find_max()

11. For $i=1$ to $\mathrm{n}$ repeat step 12 
12 .

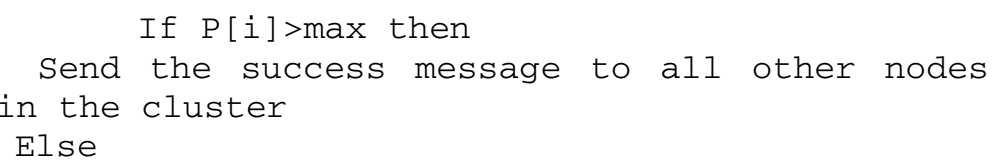

14. Aggregate collected data and send to base station

\title{
Prob_Broadcast(P[ ],n).
}

\author{
For every node of cluster i do- \\ If cluster id matches with the cluster id \\ of neighbor node $j$ then \\ Send the probability value to node $j$
}

Find_max( ) .

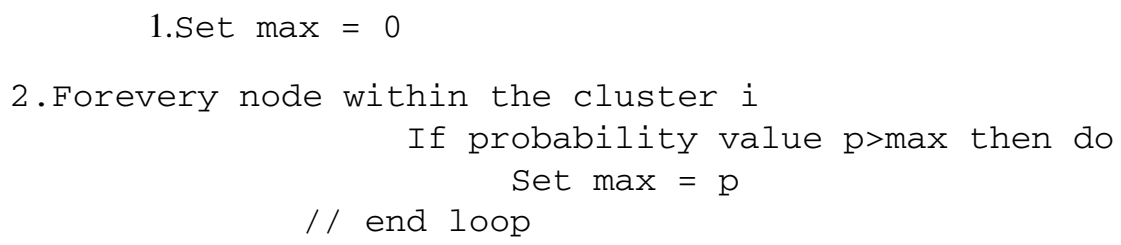

\subsection{Case Study}

In the above figure, the base station divides the network into different levels and there are different numbers of clusters in each level. The black nodes are the cluster heads of the respective clusters. Each cluster head node calculates its weight value $\mathrm{W}_{\mathrm{t}}$ except the cluster head nodes of the highest level or LEVEL 4. The weight values of the cluster head nodes of level 3 are 2.4, 2.47 and 2.3 and their distance from the base station are 255unit, 240unit and 245unit respectively and thus the node with the highest weight value that is the cluster head node having the weight value 2.47 is chosen as the leader node of level 3 and is marked in the figure with a rectangle. Every cluster head node of level 4 forwards their data to the leader node of level 3. In level 2 the distance from the base station of the cluster head nodes are 100unit, 130unit and 98unit and their weight values are obtained as 3, 3.5 and 3.2 respectively. It is observed that the node having distance from the base station 98unit has a lower weight value than the node having distance from the base station 100unit, due to having lower residual energy. Thus the node having greater weight value is chosen as the leader node to increase the lifetime of the network. Forwarding of data to the leader nodes of the next lower level continues till level 1 is reached. All the cluster head nodes of level 1 directly forward the data to the base station. 


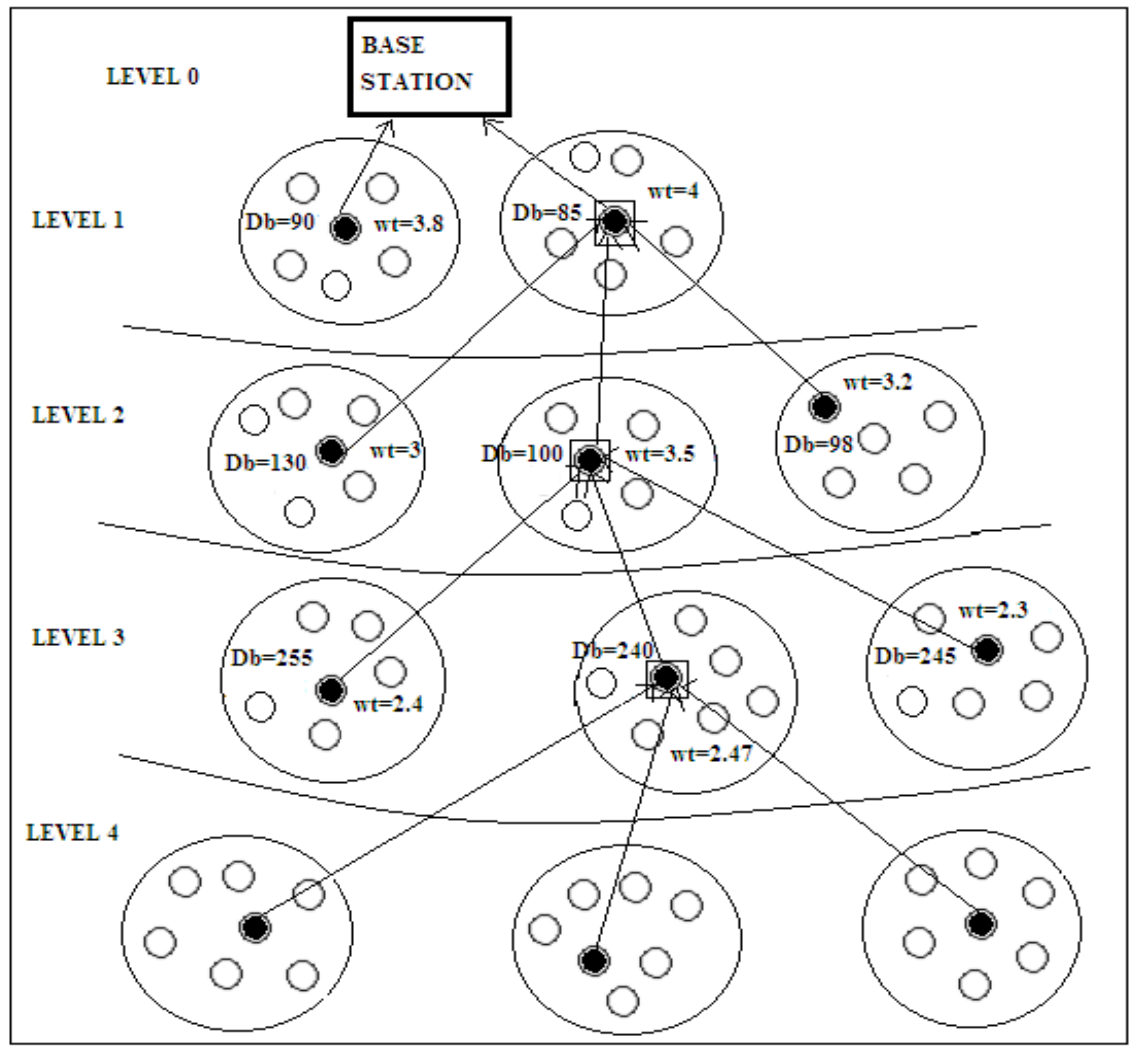

Fig. 1. Sending aggregated data through different levels

\section{Simulation Result}

To analyze the performance of the algorithm, a network with 20 nodes is created, which are distributed equally in four clusters residing in two different levels. The parameter list is given below-

Table 2. Parameter list

\begin{tabular}{|l|l|}
\hline \multicolumn{1}{|c|}{ Parameters } & \multicolumn{1}{c|}{ Description } \\
\hline Network size & 100 nodes \\
\hline Initial energy & 5000J per node \\
\hline MAC Protocol & IEEE 802.15.4 \\
\hline Power consumption & $\begin{array}{l}\text { Equivalent to packet size } \\
\text { and distance }\end{array}$ \\
\hline Number of rounds & At least 6 \\
\hline
\end{tabular}

The initial power of every node is considered 500 units. The size of each packet of data is taken as $1 \mathrm{~KB}$. After every round the total reduction in energy of the network 
and the total number of dead nodes is calculated. The result obtained is compared with that of Energy Efficient Clustering Algorithm (EECA) [3] which proves that after 6 th round more nodes are dead for EECA with compare to PACBR.

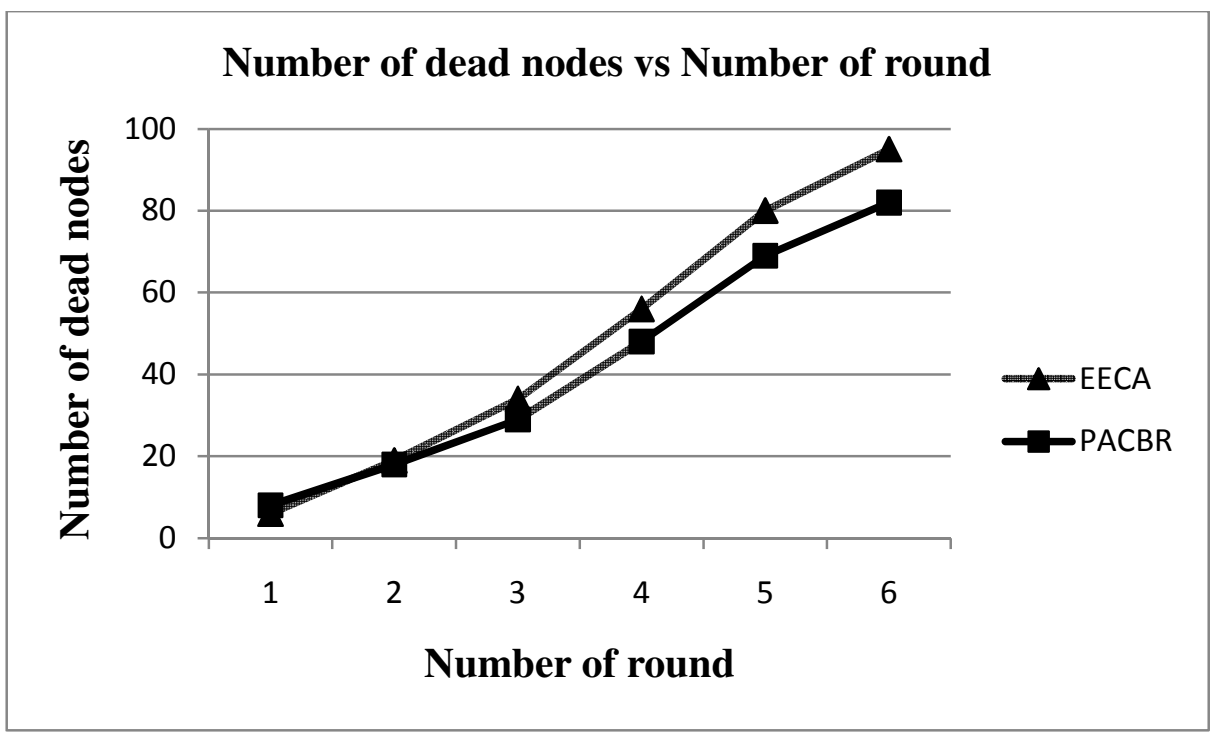

Fig. 2. Number of dead nodes in the network after each round

Again after each round the average residual energy of the network is measured for both the algorithms and has plotted as follows-

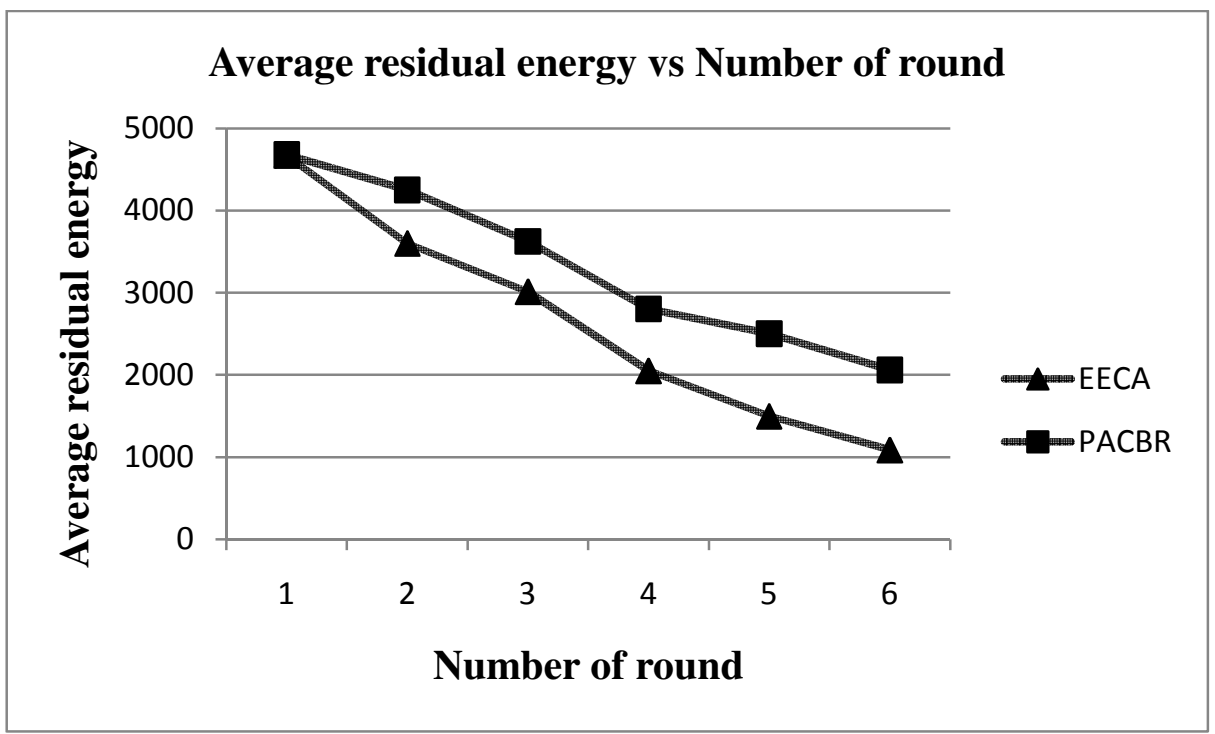

Fig. 3. The total loss of energy after each round 
The above figure shows that after 6th round the algorithm EECA [3] lost more energy than PACBR. Thus the proposed algorithm PACBR can send the aggregated data to the base station in an energy efficient way and increases network lifetime.

\section{Conclusion}

The proposed algorithm Power Aware Cluster Based Routing (PACBR) protocol for Wireless Sensor Network takes care of the longevity of the network. It divides the network into different levels and each level contents of multiple clusters. Each cluster has a cluster head. The cluster heads collects sensed data from other nodes, aggregate that and sends that to the base station in an energy efficient way. The simulation results prove that the algorithm works more efficiently than other existing algorithms and also increases network lifetime.

\section{References}

1. Geetha, V., Kallapur, P.V., Tellajeera, S.: Clustering in Wireless Sensor Networks: PerformanceComparison of LEACH\& LEACH-C Protocols Using NS2. In: 2nd International Conference on Computer, Communication, Control and Information Technology (C3IT 2012), February 25-26, vol. 4, pp. 163-170. Elsevier Journal (2012)

2. Xinhua, W., Sheng, W.: Performance Comparison of LEACH \& LEACH-C Protocols by NS2. In: 9th International Symposium on Distributed Computing and Applications to Business Engineering and Science (DCABES) (2010)

3. Sha, C., Wang, R., Huang, H., Sun, L.: Energy EfficientClustering Algorithm for data aggregation in WSN. Elsevier Journal, the Journal of China Universities of Posts and Telecommunications (December 2010)

4. Chamam, A., Pierre, S.: On planning of WSNs: Energy Efficient Clustering under the joint Routing and coverage constraint. IEEE Transactions on Mobile Computing 8(8) (August 2009)

5. Kumar, D., Aseri, T.C., Patel, R.B.: Energy Efficient Heterogeneous Clustered scheme for Wireless Sensor Network. Computer Communication 32(4), 662-667 (2008)

6. Guo, S., Zheng, J., Qu, Y., Zhao, B.H., Pan, Q.K.: Clustering and multi-hop routing with power control in wireless sensor networks. The Journal of China Universities of Posts and Telecomunications 14(1) (March 2007)

7. Tang, S., Li, W.: QoS supporting and optimal energy allocation for a cluster based wireless sensor network. Computer Communication Journal 29(13-14), 2569-2577 (2006)

8. Younis, O., Fahmy, S.: HEED: a hybrid, energy-efficient, distributed clustering approach for ad hoc sensor networks. IEEE Transactions on Appears in Mobile Computing 3(4) (October-December 2004) ISSN: 1536-1233

9. Cheng, H., Yang, G., Hu, S.: NHRPA: a novel hierarchical routing protocol algorithm for wireless sensor networks. The Journal of China Universities of Posts and Telecommunications 15(3), 75-81 (2008) 
10. Lindsey, S., Raghavendra, C.S.: PEGASIS: power efficient gathering in sensor information systems. In: Proceedings of the IEEE Aerospace Conference, Big Sky, Montana (March 2002)

11. Heinzelman, W.B., et al.: An application-specific protocol architecture for wireless microsensor networks. IEEE Transactions on Wireless Communications 1(4), 660-670 (2002)

12. Heinzelman, W.R., Chandrakasan, A., Balakrishnan, H.: Energy Efficient Communication Protocol for Wireless Microsensor Networks. In: Proceedings of the 33rd Hawaii International Conference on System Sciences (2000) 